\title{
Una arquitectura y mapa de ruta para un método de detección de debris flows y fenómenos similares en ríos, mediante una red de sensores inalámbricos
}

\author{
An architecture and a roadmap for a detection \\ method for debris flows and similar phenomena \\ in rivers, using a wireless sensor network
}

Oscar Vargas Fallas'

Francisco Torres Rojas ${ }^{2}$

Fecha de recepción: 9 de marzo del 2012

Fecha de aprobación: 26 de setiembre del 2012

Vargas, O; Torres, F. Una arquitectura y mapa
de ruta para un método de detección de
debris flows y fenómenos similares en ríos
mediante una red de sensores inalámbricos.
Tecnología en Marcha. Vol. 26, N ${ }^{\circ}$ I. Pág 54-65

I Ingeniero en Electrónica, Instituto Costarricense de Electricidad. Estudiante del Programa de Maestría en Computación del Instituto Tecnológico de Costa Rica. Correo electrónico: ovargasf@ice.go.cr

2 Doctor en Ciencia de la Computación. Profesor e investigador de la Escuela de Ingeniería en Computación, Instituto Tecnológico de Costa Rica. Correo electrónico: torres@ic-itcr.ac.cr 


\section{Palabras clave}

Redes de sensores, debris flows, sistemas de alerta temprana.

\section{Resumen}

Los debris flows y flujos hiperconcentrados, conocidos popularmente como cabezas de agua, aluviones o avenidas de lodo, son fenómenos muy destructivos que afectan a poblaciones ubicadas en márgenes de ríos. Los sistemas utilizados para detectar este tipo de eventos se basan en instrumentación fija en puntos determinados del río o a través de mediciones remotas e indirectas, por lo cual los datos que suministran son limitados: no brindan información en tiempo real sobre la posición o velocidad del evento más allá de los puntos de monitoreo. En este artículo se propone una arquitectura para la detección de debris flows y flujos hiperconcentrados en cuencas de ríos, por medio de una red inalámbrica de sensores móviles. La arquitectura propuesta es distribuida y descentralizada, pues no depende de un ente coordinador central para decidir sobre la presencia o ausencia del fenómeno. También se plantea un mapa de ruta de los problemas que deben superarse, con el fin de hacer posible la implementación de esta arquitectura en un sistema de detección concreto para los ríos.

\section{Key words}

Wireless sensor networks, debris flows, early warning systems.

\begin{abstract}
Debris flows and hiperconcentrated flows are very destructive phenomena that cause damage, injures and deaths to property and persons that live near rivers. Existing warning systems for these phenomena are based on fixed instrumentation on certain points of the river, or on remote, indirect measurements. As a result, the information that they provide is limited, neither real-time data about the advance of the flow trough the river, nor its velocity outside the fixed monitoring points is available. In this article, an architecture for a debris flows and hiperconcentrated flows detection method based on a wireless sensor network is proposed. This architecture is distributed and decentralized, since it does not depend on a central coordinator to decide on the presence or absence of the phenomenon. A roadmap for further research is also proposed, in order to solve challenges and problems that must be overcome in order to make possible an implementation of this architecture in a real world detection system.
\end{abstract}

\section{Introducción}

Los debris flows y flujos hiperconcentrados, conocidos popularmente como cabezas de agua, aluviones o avenidas de lodo, son fenómenos muy destructivos que afectan a poblaciones ubicadas en márgenes de ríos. Por ejemplo, el debris flows ocurrido en Calle Lajas de Escazú, en Costa Rica en 20 I 0, cobró la vida de 24 personas (López, 20 I 0), o la tragedia en Alto Loaiza de Orosi, que dejó siete víctimas humanas y 24 familias sin hogar (Murillo, 2003). Los flujos originados a raíz del terremoto de Cinchona ocasionaron la destrucción de seis puentes, daños en la planta hidroeléctrica Cariblanco y la muerte de una persona (Alvarado, 2010).

Los sistemas que se han utilizado para detectar este tipo de eventos se basan en instrumentación fija en puntos determinados del río o en mediciones remotas e indirectas, por lo que los datos que suministran son limitados y no brindan información en tiempo real sobre el avance o velocidad del evento más allá de los puntos de monitoreo (Lee et al., 20 I0). Estos sistemas se han basado en distintas técnicas. Una de ellas es la colocación de alambres electrificados a cierta altura cruzando el río, de modo que al ocurrir el evento, este rompe el alambre y por lo tanto se interrumpe el paso de la corriente eléctrica. Otros sistemas más sofisticados utilizan sensores ultrasónicos o de radar para medir el nivel del río en ciertos puntos. También se han utilizado mediciones indirectas, por medio de geófonos y sismómetros, para detectar las vibraciones causadas por los flujos. Otros, los predicen a partir del monitoreo de las condiciones hidrometereológicas de una región (Arattano \& Marchi, 2008). 
Una de las tecnologías en desarrollo que promete nuevas soluciones para sistemas de alerta temprana de desastres naturales es la de Redes Inalámbricas de Sensores (RIS). Estas redes consisten en pequeños dispositivos, llamados nodos, con capacidad sensorial y de procesamiento de datos, que se enlazan mediante una red inalámbrica ad-hoc, es decir, creada espontáneamente en el sitio.

Como parte de una investigación en desarrollo, en este artículo se propone desarrollar y evaluar un método de detección basado en una red inalámbrica de sensores móviles. La construcción y colocación de estos sensores en el cauce del río será tal que serán arrastrados por la corriente al ocurrir un debris flows. Al ser arrastrados, estos detectarán la presencia de un fenómeno de este tipo y la reportarán a otros nodos río abajo, los cuales retransmitirán esta información hasta los dispositivos receptores encargados de alertar a la población. Al estar en contacto directo con el fenómeno y no depender de una infraestructura de comunicación fija, un sistema de detección basado en este método podría ser más confiable y de menor costo de desplegar y mantener que los métodos que existen actualmente.

El artículo está estructurado de la siguiente forma: en la presente sección se introduce la idea de la propuesta. En la sección 2 se da un breve fundamento teórico y definiciones sobre los fenómenos de debris flows y flujos hiperconcentrados, así como sobre RIS. En la sección 3 se hace referencia a trabajos previos en temas relacionados. En la sección 4 se propone una arquitectura de solución y se detallan algunos de sus aspectos. En la sección 5 se plantea un mapa de ruta para orientar el trabajo futuro que se realice en este tema. Por último, se brindan algunas conclusiones sobre lo aquí propuesto.

\section{Conceptos básicos}

\section{Debris flows y fenómenos relacionados}

Los debris flows' y flujos hiperconcentrados son movimientos de material que ocurren en torrentes montañosos. Se componen de agua con una alta concentración de sedimentos de partículas de

I Debido a que en idioma español no existe una nomenclatura técnica estándar para estos términos (ya que varían según la región), se utilizan términos en inglés como debris flow. diversos tamaños y otros materiales, se mueven a muy alta velocidad por lo que resultan altamente destructivos (Arattano \& Marchi, 2008). Estos eventos pueden ocurrir tras precipitaciones intensas, en las que el flujo de material excede la capacidad del canal y devastan la tierra en la vecindad del río (Davies, 1986).

\section{- Debris flows}

Consisten en una mezcla saturada de detritos, entre ellos roca, material orgánico y agua, que se mueve en dirección descendente por efecto de la gravedad. Contienen al menos un $50 \%$ de sedimentos (Ritchie \& Gates, 2007, Vallance, 2000).

\section{- Flujos hiperconcentrados}

Consisten en una mezcla no uniforme de detritos y agua, movida por gravedad. El contenido de agua es mayor que el de un debris flows, con un contenido de detritos del 20\% al 60\%. Aunque conserva características fluviales, arrastra gran cantidad de sedimentos (Vallance, 2000).

\section{Redes Inalámbricas de Sensores (RIS)}

Las RIS consisten en pequeños dispositivos con capacidad sensorial y de procesamiento y bajo consumo de energía; enlazados mediante redes creadas de manera ad-hoc, es decir, organizadas de forma autónoma entre los dispositivos y con capacidad de reconfigurarse dinámicamente en caso de que la red cambie, por ejemplo, al quedar algún nodo fuera de ella debido al agotamiento de su fuente de energía o al ser destruido. En este tipo de redes, la información recolectada en cualquiera de sus nodos es enrutada a través de otros nodos de la red hacia uno o varios sumideros o sinks que la recogen y transfieren a sus usuarios, como se muestra en la figura I.

Debido a sus características, este tipo de redes resulta de gran utilidad para la observación de fenómenos ambientales sobre un área geográfica extendida. El hecho de que puedan funcionar de forma desatendida, con mantenimiento mínimo y en entornos hostiles las hace idóneas para aplicaciones de monitoreo de amenazas ambientales, en particular para sistemas de predicción y detección.

Ya se han propuesto aplicaciones basadas en RIS para la alerta temprana de amenazas naturales, como los deslizamientos (Anandarajah et al., 2005; 


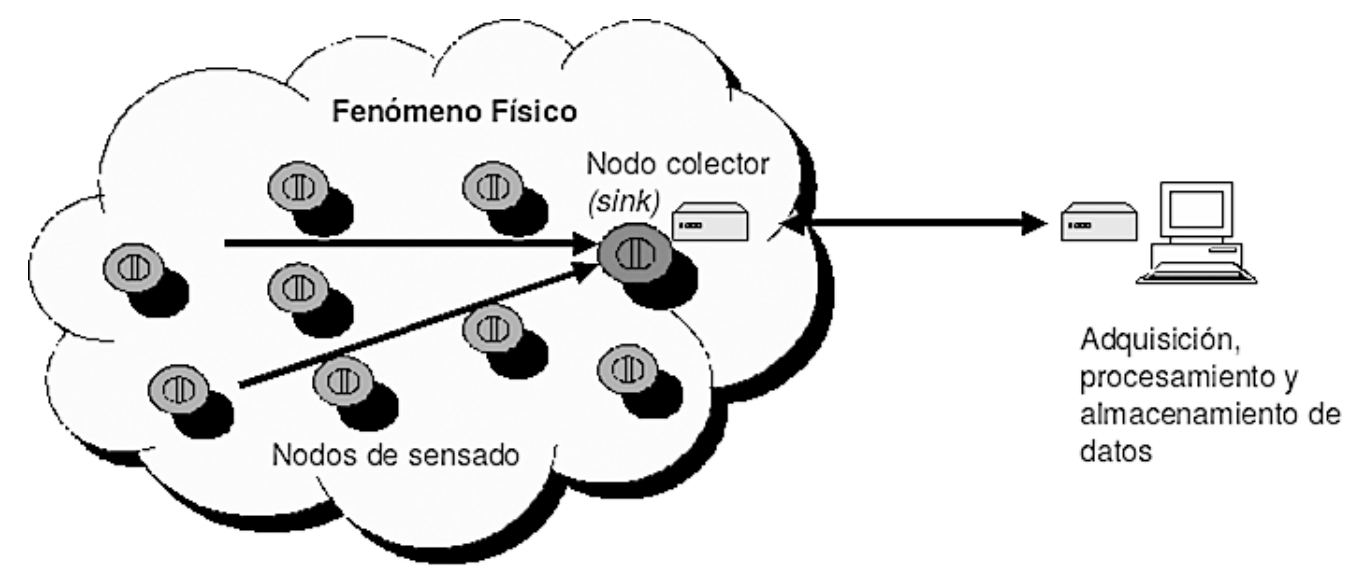

Figura I. Esquema de una red de sensores inalámbricos

Arnhardt et al., 2007; Sheth et al., 2005), inundaciones (Blyth, 1997) y avalanchas de nieve (Henderson, 2004).

\section{Antecedentes}

La idea de utilizar sensores móviles arrastrados por la corriente para detectar este tipo de eventos fue propuesta y desarrollada por Lee et al. (2008, 2009a,b, 20I0), quienes plantean colocar un conjunto de dispositivos sensores denominados Insider en el lecho del río para que sean arrastrados por la corriente en caso de un debris flows. Estos dispositivos miden parámetros internos del evento y transmiten la información a dispositivos receptores ubicados en la margen del río, denominados Coordinator, los cuales se encargan de reenviarla al centro de recolección de datos.

Anteriormente, Hanisch (2003) había propuesto el uso de un dispositivo similar a una roca, para ser arrastrado en debris flows dotado de instrumentación para registrar variables importantes del evento para su posterior extracción y análisis.

Zostrich Geotechnical (2003) anunció el desarrollo y solicitud de una patente para un sistema de alerta temprana basado en dispositivos sensores inalámbricos, dotados de una celda solar, radio, acelerómetro, GPS y batería; sin embargo, no ha reportado avances ni pruebas realizadas o prototipos del mismo.

Tanto Lee et al. (2008) como Zostrich Geotechnical (2003) proponen esquemas centralizados, en los cuales los datos recabados por cada nodo sensor en última instancia llegarán a un ente central que tiene pleno conocimiento de lo que sucede en el sistema y es el encargado de determinar el estado del mismo y decidir si se declara una alarma.

A diferencia de estos trabajos previos, proponemos un esquema descentralizado, donde la decisión sobre la presencia o ausencia de un evento se hace de forma distribuida entre los distintos nodos sensores. Luego, esta decisión es propagada a otros nodos río abajo, para que la población potencialmente afectada pueda recibirla.

\section{Arquitectura propuesta}

\section{Descripción general}

Se propone un esquema para la detección mediante una RIS constituida por nodos móviles. El suministro de energía de cada sensor está a cargo de su propia batería, lo que le permitiría operar incluso al ser arrastrado. Debido a que las plataformas de hardware utilizadas para el desarrollo y construcción de nodos sensores se caracterizan por tener un consumo muy bajo de potencia (del orden de microamperios), el uso de sensores alimentados por baterías se convierte en una alternativa viable, pues los sensores podrían funcionar hasta por tres años sin requerir un cambio de batería (Polastre et al., 2005).

El método sigue un paradigma de detección descentralizado, que no depende de una entidad central 
para declarar la alerta, sino que esta se genera de forma cooperativa entre los distintos nodos de la RIS. Una característica importante de este método es que no requiere hardware o instrumentación especializada para medir alguna variable física del entorno. En la figura 2 se ilustra de forma general este método de detección.

Inicialmente, los sensores se colocan dentro del cauce del río y a la orilla de este, como se aprecia en la figura 2a. Al ocurrir un evento como un debris flow este arrastra los sensores que se encuentran a su paso (figura 2b y 2c). Los sensores detectan de forma distribuida el cambio en la configuración de la red y deciden por consenso que están en presencia de un evento que debe reportarse (figura $2 \mathrm{~d}$ ), por lo que notifican a los sensores ubicados río abajo, que a su vez propagarán esa alerta hacia el resto de sensores en dirección descendente (figura 2e) hasta que todos los nodos de la red tengan conocimiento del evento (figura 2f) y alerten a la población en peligro.

\section{Topología}

Considérese el caso simple en el que se observa un segmento del cauce de un río, sin sus afluentes. Los sensores se distribuyen a lo largo de este segmento (dentro del cauce y a la orilla), como se ilustra en la figura $3 \mathrm{a}$.

Es necesario que el área cubierta por los sensores incluya las partes altas del río, lo que permitiría detectar el debris flow con la mayor anticipación posible. También debe incluir las partes intermedias del mismo, así como las contiguas a las zonas pobladas que se requiere alertar, pues debe existir un camino para que la alerta se propague desde la zona de detección hasta los receptores de la misma. Esta disposición de sensores produce como resultado una topología de malla, cuyo tejido emula

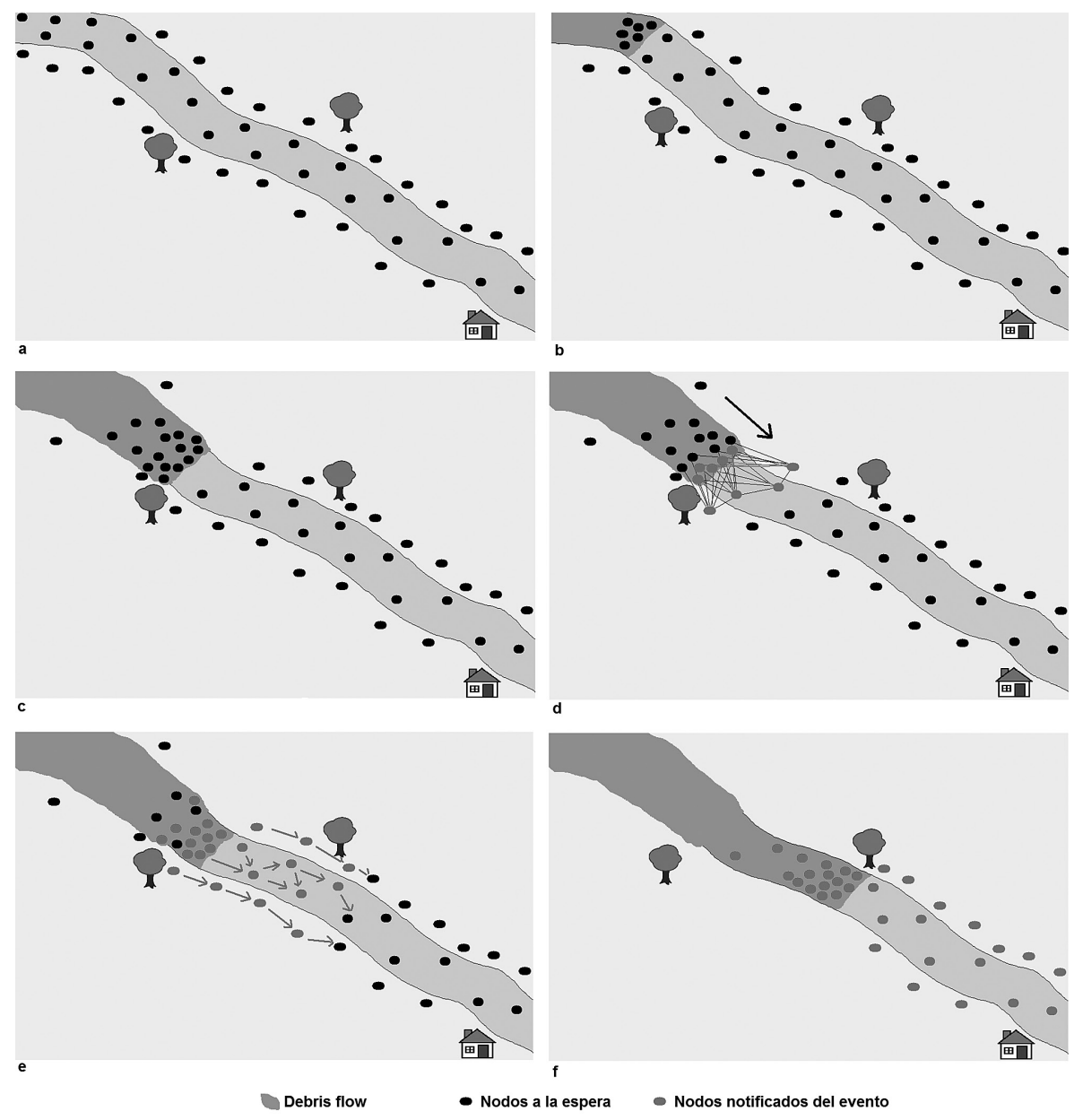

Figura 2 Descripción del método de detección 


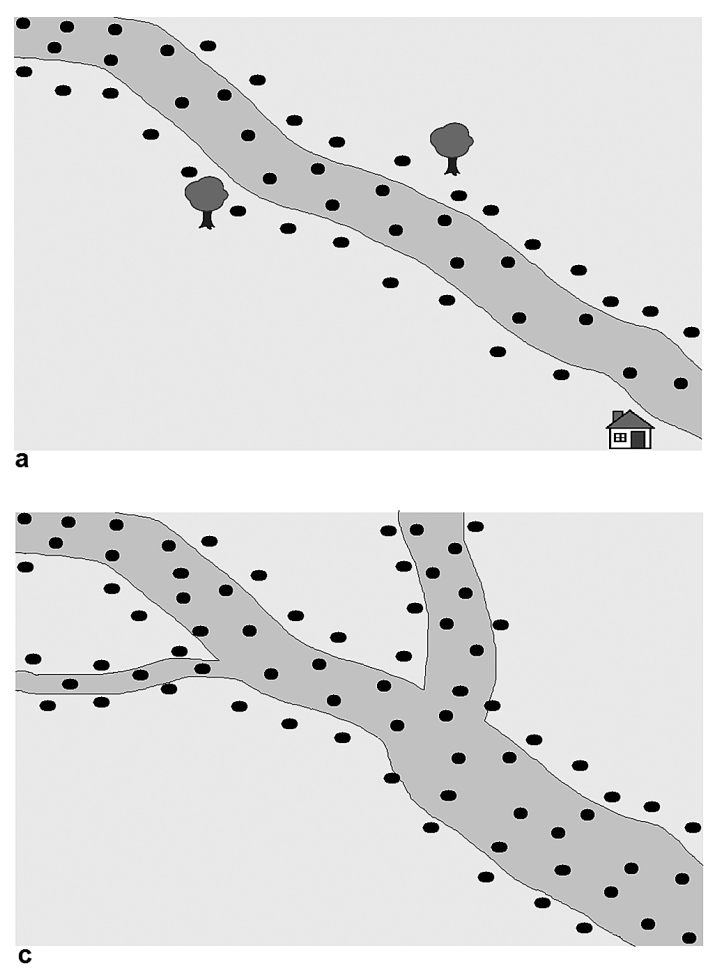

Figura 3. Topología de la red de sensores el cauce del río, como se ilustra en la figura 3b. En dicha figura, cada nodo en la red está representado por un punto; las líneas representan enlaces entre nodos los suficientemente cercanos como para establecer una comunicación bidireccional directa entre ellos.

Una vez considerado el caso simple, correspondiente a observar un segmento de río sin contemplar sus afluentes, se puede considerar el caso más general, en el cual se monitorea un río principal junto con sus afluentes. La red resultante tiene la disposición mostrada en la figura 3c. Al igual que el caso anterior, la topología resultante emula el cauce del río, esta vez conformado por su cauce principal y alimentado por varias afluentes. Nótese que la misma presenta ramificaciones, de manera similar a la estructura de un árbol, como se muestra en la figura 3d.

\section{Modelo de trasiego de datos}

De acuerdo con la taxonomía propuesta porTilak et al. (2002), el modelo de trasiego de datos en la red de sensores que se propone es de trasiego desencadenado por evento, pues solo en caso de detec-

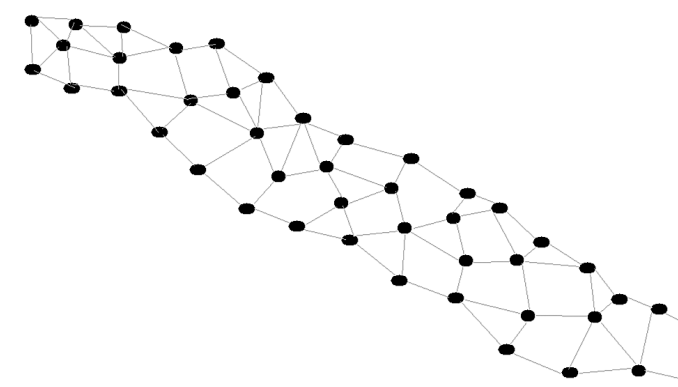

b

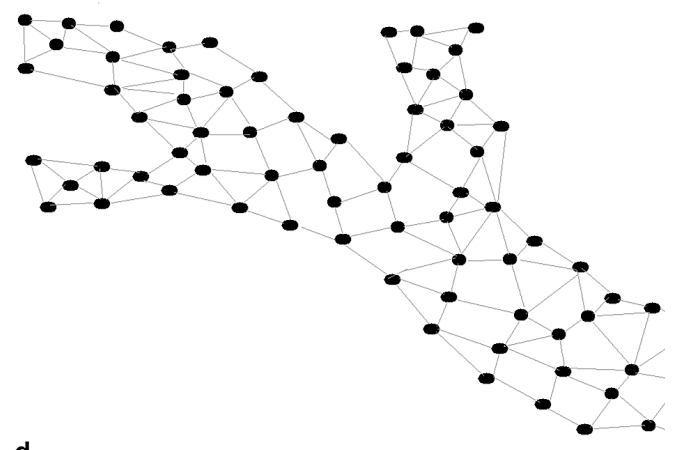

d

tarse un debris flow o un evento similar se reportan datos a los usuarios de la red. No se envían datos de forma periódica ni los usuarios tienen capacidad para hacer consultas sobre el estado del área observada. Este modelo de trasiego de datos es natural para la arquitectura propuesta y para el propósito de la RIS, ya que a los usuarios de la red solo les interesa ser notificados en caso de un evento, además de que en este planteamiento no se contempla que los sensores midan variables del entorno, que puedan ser consultadas por los usuarios. Cualquier otro modelo de trasiego de datos tendría un impacto mayor e innecesario sobre el consumo de energía, y por ende, sobre el tiempo de vida de la RIS.

\section{Sensores}

Estos son los elementos físicos encargados de detectar la presencia del debris flow al ser arrastrados por él. Cada uno estará integrado por un microcontrolador o microprocesador empotrado (MCU), radio transmisor-receptor que lo comunica con otros sensores, suministro de energía y encapsulado físico, como se ilustra en la figura 4. 


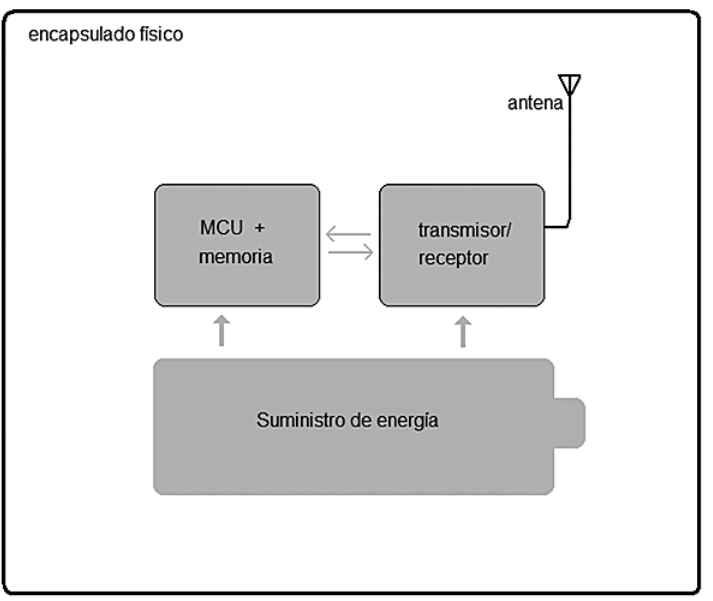

Figura 4. Diagrama de bloques de un nodo sensor

Los sensores deben ser del menor costo posible, pues deben colocarse muchos de ellos a lo largo del cauce del río. Además, los sensores no son reutilizables, pues no resulta práctico recuperarlos luego de un evento. El diseño y la construcción física de los mismos debe ser tal que durante el comportamiento normal del río permanezcan estáticos donde fueron ubicados y al ocurrir un evento sean arrastrados por él. Además, deben soportar las condiciones físicas ambientales del entorno en que serán ubicados, durante el tiempo de vida requerido. En esta propuesta no se requiere que los sensores cuenten con instrumentación o hardware especializado para medición de variables físicas. La estrategia de detección está basada en detectar la proximidad de otros nodos. Esta estrategia no excluye el uso de acelerómetros u otros elementos sensores de movimiento, pero la estrategia aquí propuesta no presupone su presencia.

El suministro de energía es un factor determinante, pues una vez que esta se agote, el nodo será inoperante en la red. Esto debe ser tomado en cuenta para determinar el tiempo de vida de la misma. Aunque existen métodos para extraer energía del medio ambiente (a esta práctica se le conoce como energy harvesting), estos pueden elevar el costo de cada sensor y hacerlos susceptibles al vandalismo; por lo que en principio se propone depender exclusivamente de baterías. Esto es posible gracias a que las plataformas de hardware utilizadas para motes o nodos de RIS tienen un consumo de energía muy bajo, pensado justamente para aplicaciones en que un nodo sensor debe funcionar hasta por tres años con solo un paquete de baterías como suministro de energía (Polastre et al., 2005).

\section{Estrategia de detección}

La estrategia de detección propuesta es la siguiente: una vez desplegada, la red construirá de forma distribuida un mapa de la misma, de forma que cada sensor conozca su posición dentro de la red, cuáles nodos están ubicados río arriba y cuáles río abajo. Este mapa se actualizará de forma periódica para incorporar cambios que ocurran en la topología de la red.

Considérese un sensor de la red en ausencia de un evento. Este podrá recibir mensajes periódicos directamente de sensores cercanos, con una señal de intensidad más o menos fuerte. También podría recibir mensajes de otros sensores ubicados un poco más lejos, entonces la intensidad de la señal recibida sería menor, por lo que el enlace sería más débil y podría ser intermitente, según las condiciones ambientales.

En presencia de un debris flow, los sensores ubicados río arriba, donde está ocurriendo el evento, irán siendo arrastrados. Cuando se acerquen al sensor en cuestión, este podrá recibir directamente los mensajes provenientes de ellos. ${ }^{2}$ Como en el mapa de la red, que internamente almacena el nodo, la ubicación de estos sensores estaba situada río arriba, se puede tomar esto como un indicio de que se aproxima un debris flow.

Debido a que nodos vecinos (especialmente río arriba) deberían observar un comportamiento similar, una vez que se sospecha que se aproxima un debris flow, el nodo envía mensajes a los nodos vecinos, con lo que se activa un protocolo para decidir de forma distribuida si realmente se aproxima un debris flow o no. En caso de resultar positiva, se generará de forma consensuada una alerta que cada nodo difundirá a todos sus vecinos, que a su vez la difundirán río abajo hasta alcanzar los dispositivos receptores de la misma.

2 Debido a que el sensor que emite se encuentra sumido en un debris flow, las condiciones serán adversas para la comunicación vía RF; por lo que no es realista esperar una comunicación clara e ininterrumpida hacia los nodos que vayan apareciendo río abajo. Sin embargo, para efectos de este trabajo se supondrá que, de una serie de mensajes emitidos por el nodo, existe una alta probabilidad de que al menos uno será recibido por otro nodo ubicado río abajo. 


\section{Estimación de posición y velocidad del fenómeno}

Volviendo al sensor hipotético utilizado en el ejemplo anterior, al recibir mensajes de los nodos que se aproximan hacia él río arriba, arrastrados por el flujo, puede sospechar que el flujo se aproxima hacia el nodo. Una vez alcanzado y arrastrado por el debris flow, el sensor se encuentra ahora en una trayectoria descendente por el cauce del río, por lo que podría captar señales emitidas por nodos ubicados río abajo que anteriormente no captaba.

Así pues, se puede acotar el instante $t$ en el tiempo en el que el debris flow o evento de interés alcanzó al sensor en cuestión: $t$ es posterior al intervalo en que se empiezan a recibir mensajes de nodos que vienen con el flujo y anterior al intervalo en que detecta nodos ubicados río abajo.

Si se conoce la ubicación del sensor en cuestión (o bien, existe una buena aproximación de ella), entonces se puede aproximar la posición del flujo en el instante $t$, como cercano a la posición del sensor. Si se conoce la ubicación de otros sensores cercanos y estos también resultan arrastrados, es posible también aproximar la velocidad del flujo en ese trayecto del río. Esta estimación o aproximación de la velocidad, que se calcularía de forma automática en los dispositivos receptores, puede dar un dato aproximado a las personas ubicadas en la zona de peligro de cuánto tiempo tienen para evacuarla.

Para poder utilizar este esquema de estimación de posición y velocidad del flujo, debe conocerse la ubicación, exacta o aproximada, de cada sensor, antes de que ocurra el evento. Esto puede hacerse de forma manual, registrando las posiciones de cada sensor al desplegar la red, o bien, siguiendo un algoritmo de localización como los propuestos por Kwon \& Song (2008), Kwon \& Agha (2008), Park et al. (2008) y Klingbeil \& Wark (2008). Aunque es técnicamente posible dotar a cada sensor con un módulo de GPS, esto encarecería considerablemente el sensor y deterioraría la vida del mismo al aumentar el consumo de energía.

\section{Propagación de la alerta}

Una vez declarada la alerta, ésta será propagada hacia los sensores ubicados en el río aguas abajo. Para esto, cada sensor que genere o reciba una alerta la enviará a todos sus vecinos en esa dirección. Este procedimiento se ilustra en la figura 5 y se detalla a continuación:

I. Inicialmente los sensores ubicados en la parte alta del río son arrastrados por el flujo (figura $5 a)$.

2. Los sensores ubicados en la frontera entre el debris flow y el cauce normal del río detectan y declaran la presencia del evento (figura 5b).

3. Los sensores que han detectado el evento notifican la alerta a los sensores vecinos ubicados en dirección río abajo (figura 5c).

4. Al recibir la alerta, cada uno de los sensores que la reciba la reenviará a todos sus vecinos hacia abajo (figuras $5 d$ y $5 e$ ).

5. Finalmente, toda la red tendrá conocimiento de la presencia del evento (figura 5f).

Recepción de la alerta

Mediante el esquema de propagación propuesto, la alerta se diseminará al resto de nodos en la red ubicados río abajo. A la orilla del río, en las zonas pobladas, donde se requiere alertar en caso de un evento, se instalarán los receptores de la alerta. Estos receptores contarán con una interfaz de radio compatible con los del resto de sensores en la red; por lo que formarán parte de la red de sensores. A diferencia de los nodos sensores, estos no se construirán para ser arrastrados por el río, sino que deberán construirse de tal forma que puedan ubicarse cerca de la margen del río para recibir la alerta y contar con un mecanismo de alerta a la población cercana, por ejemplo, una sirena.

\section{Mapa de ruta de problemas que se deben resolver para construir un sistema de detección basado en la arquitectura propuesta}

Actualmente, se está trabajando en proponer un algoritmo para la detección de eventos, así como en hacer una evaluación del mismo por medio de simulación. También se hará una implementación en un prototipo, como prueba de concepto.

Para hacer posible la construcción de un sistema de detección basado en este método, deben resolverse varios problemas, que en esta sección se identifican, con el objetivo de orientar el trabajo futuro. Estos 

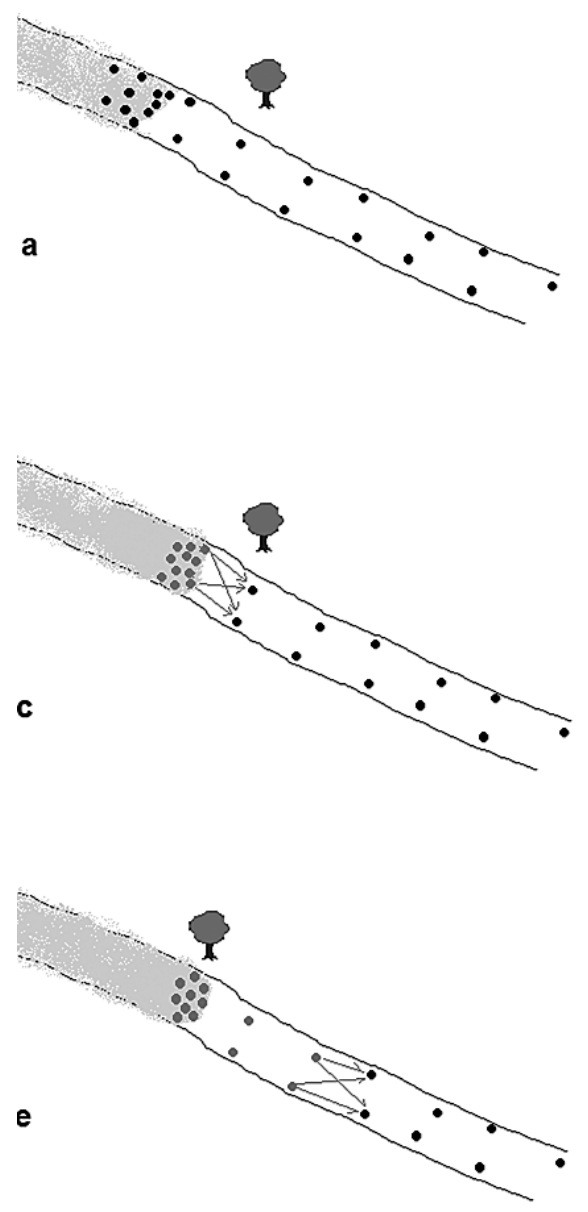

- Nodo a la espera

- Nodo alertado
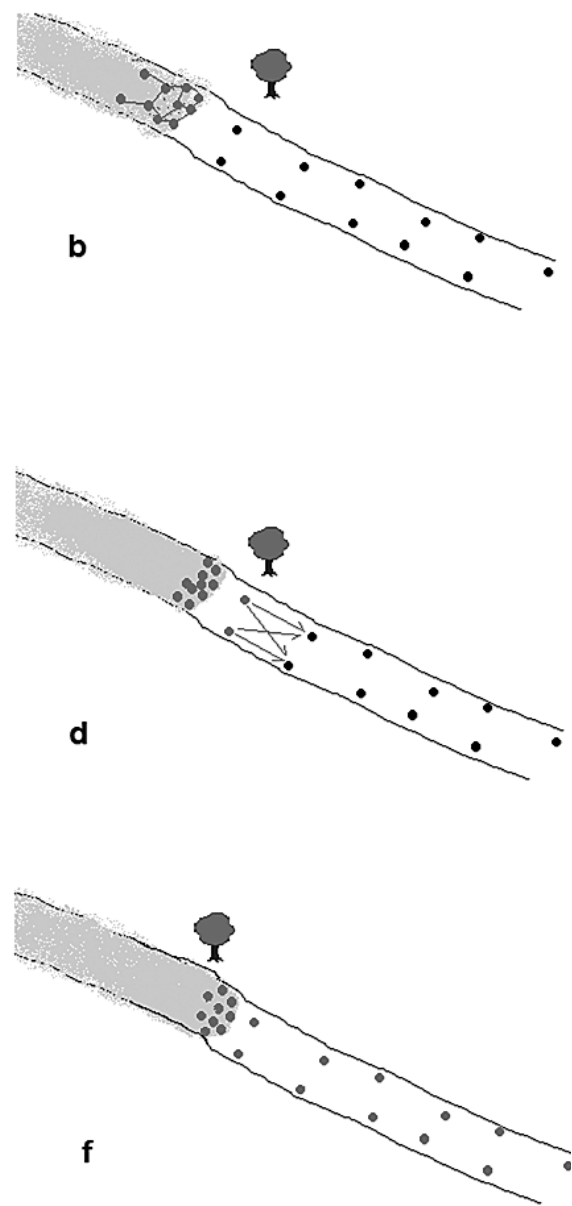

$\longrightarrow$ Mensaje de alerta

Figura 5 Propagación de la alerta

retos o problemas se han clasificado en dos grandes grupos: problemas tecnológicos y problemas no tecnológicos.

\section{Problemas tecnológicos}

\section{Aspectos de conectividad y radiofrecuencia:}

Se incluyen aquí los problemas relacionados con propagación de señal de radiofrecuencia y la conectividad de la red. Este es un aspecto de mucha importancia en esta aplicación, pues los dispositivos emisores y receptores pueden encontrarse inmersos en una mezcla de lodo, detritos y agua; por lo que se hace necesario estudiar su comportamiento en estas condiciones.

En particular, deben estudiarse los siguientes aspectos: a) Modelo de propagación de señal en un escenario de debris flow o flujo hiperconcentrado: debe trabajarse en la adopción de un modelo formal de propagación de radio para los nodos de la RIS, que tome en cuenta las características relevantes de la topografía de un río, así como las condiciones físicas del entorno, tanto en régimen estacionario como durante un evento.

b) Aspectos de conectividad de la red: con respecto a este punto y basado en el modelo de propagación anteriormente mencionado, debe estudiarse cuál debe ser la separación óptima entre sensores, de tal forma que se tenga un balance entre la potencia de transmisión para que el consumo de energía sea mínimo y el número de sensores que deben colocarse en un río para que este quede cubierto. 


\section{Plataforma de hardware y software}

La plataforma de hardware es un diseño de referencia de la arquitectura del hardware (electrónica) del sensor: microprocesador o microcontrolador, radio transmisor-receptor, interfaz de entradas/salidas, etc.

Existen tanto plataformas comerciales como también diseños de referencia abiertos, que se pueden adoptar para esta iniciativa, como por ejemplo, Berkeley Mote (Ruiz-Sandoval et al., 2006). También cabe la posibilidad proponer un nuevo diseño para este sistema.

La plataforma de software incluye el sistema operativo, servicios, bibliotecas y middleware, que permiten el desarrollo del software específico para la aplicación. También incluye las herramientas o toolchain utilizadas para desarrollar aplicaciones: IDEs, compiladores, debuggers, simuladores o emuladores.

\section{Encapsulado del sensor}

El método propuesto requiere detectar el movimiento de los sensores cuando estos son arrastrados por el flujo. Esta mecánica impone un requisito físico fundamental a los sensores: su construcción debe ser tal que permanezcan inmóviles durante el curso normal del río, pero que en un evento sean arrastrados por el flujo. Además, el nodo debe soportar y proteger la electrónica interna de las condiciones físicas del entorno donde desempeñará su función. También deberá ser capaz de resistir las condiciones que se presenten al ser arrastrado por el flujo: aceleraciones, impactos, inmersión en agua y lodo. Por lo tanto, se deberá trabajar en el desarrollo de un encapsulado físico para los sensores que cumpla con estos requisitos.

\section{Desarrollo del receptor de la alerta}

Debe desarrollarse también uno o varios tipos de elementos receptores de la alerta, que la reciban de la RIS y notifiquen a la población. En su concepción más básica, estos dispositivos activarán un dispositivo de señalización, como una alarma o sirena. También es posible pensar en dispositivos más sofisticados, que también envíen alertas dirigidas a autoridades y servicios de respuesta, a través de Internet mediante redes celulares, o bien, una red de dispositivos notificadores distribuidos en la población, en la que la alarma se propague una vez sea recibida en el primer dispositivo. Puesto que estos dispositivos también formarán parte de la RIS (condición necesaria para poder recibir de ellos la alerta), también estarán basados en la misma plataforma de hardware y software de los sensores.

\section{Infraestructura de información}

Aun cuando el objetivo fundamental de un sistema de alerta es avisar del evento a la población inmediatamente afectada, también resulta deseable llevar información sobre las alertas y otros datos de la red de sensores a consumidores potenciales de esa información: servicios de emergencia y autoridades civiles, que al ser notificados de la alerta podrán despachar la ayuda lo más pronto posible al sitio indicado; encargados de gestionar la red que deseen monitorear continuamente el estado de la misma y conocer en tiempo real su capacidad de respuesta ante eventos y estimar cuándo y adónde reemplazar o agregar nuevos nodos a la red. Para esto, debe plantearse y construirse una infraestructura de información que haga posible este trasiego de información

Por infraestructura de información se entiende toda la plataforma de conectividad y software que permitiría lograr este objetivo. Se incluirían en ella: enlaces de comunicación, plataforma de red, servidores, sistemas de adquisición de datos, plataformas web y demás sistemas y aplicaciones que se adopten o se desarrollen para tal efecto. También se consideran aquí las interfaces de software que permitan llevar estos datos a otros sistemas o plataformas.

Un aspecto importante que se debe tomar en cuenta con respecto a esta plataforma de software es que debe ser interoperable, es decir, debe intercambiar información con otros sistemas y plataformas. Para esto es necesario el uso de tecnologías abiertas y que se sigan estándares relevantes a esta aplicación. Uno de ellos estrechamente relacionado para esta plataforma es la especificación Common Alerting Protocol de OASIS (2005).

\section{Problemas no tecnológicos}

\section{Gestión del sistema}

Una vez que se ponga a operar un sistema de detección, debe existir una entidad que se haga cargo del mismo: monitorear regularmente su estado para verificar que se encuentra operativo y listo para detectar un evento, dar el mantenimiento 
correspondiente a los equipos que lo requieran y reemplazar o agregar nuevos nodos a la red cuando sea necesario.

Adopción por comités de emergencias y servicios de respuesta

Un sistema como el que se plantea aquí debe formar parte de un esfuerzo integral y concertado para la gestión de los riesgos por amenazas naturales. Por ello, resulta necesario involucrar tanto a las autoridades nacionales y regionales encargadas de la gestión de este tipo de riesgos como a los comités locales de atención de emergencia y a los cuerpos de respuesta a este tipo de emergencias, para integrar este sistema de detección en sus planes de mitigación de riesgos, recibir retroalimentación sobre el funcionamiento de la red y los mecanismos de alerta y que el sistema en general sea utilizado de la mejor forma para atender las emergencias detectadas por él.

Además, es necesario involucrar, capacitar y organizar a la población para responder a las alertas que reporte el sistema. Si la población cuenta con un Comité Local de Emergencia, que contemple este tipo de amenazas, es imperativo hacerle partícipe del sistema e integrarlo a sus recursos, para que sea este comité el encargado de educar a la población en la atención de las alarmas que genere el sistema y defina los procedimientos de alerta y evacuación correspondientes.

\section{Conclusiones}

En este artículo se presenta el planteamiento de un método para la detección de debris flows y flujos hiperconcentrados en cauces de ríos, de forma distribuida y descentralizada, mediante una red de sensores. También se presenta un mapa de ruta que señala los principales retos que deben superarse para hacer posible la implementación concreta de este método en un río específico.

Actualmente se está trabajando en el planteamiento y evaluación de los algoritmos y protocolos correspondientes; también en la implementación de un prototipo físico, que sirva como prueba de concepto de este método. Los resultados que se obtengan tanto en la evaluación de algoritmos y protocolos como en la implementación del prototipo servirán para entender mejor el comportamiento de la red de sensores en condiciones reales, lo que permitirá orientar el trabajo futuro para así lograr hacer posible una implementación real de este método.

\section{Bibliografía}

Alvarado, G.E. (20 I 0). Aspectos geohidrológicos y sedimentológicos de los flujos de lodo asociados al terremoto de Cinchona $\left(M_{w}\right.$ 6,2) del 8 de enero de 2009. Revista Geológica de América Central 43: 67-96.

Anandarajah, A., Moore, K., Terzis, A. \& Wang, I.-J. (2005). Sensor Networks for Landslide Detection. Proceedings of the third international conference on embedded networked sensor systems, pp. 268-269.

Arattano, M. \& Marchi, L. (2008). Systems and Sensors for Debrisflow Monitoring and Warning. Sensors 8(4): 2436-2452. Obtenido desde: https://www.mdpi.org/sensors/papers/ s8042436.pdf

Arnhardt, C., Asch, K., Azzam, R., Bill, R., Fernandez-Steeger, T.M., Homfeld, S.D., Kallash, A. \& Walter, K. (2007). Sensor based Landslide Early Warning System - SLEWS. Development of a geoservice infrastructure as basis for early warning systems for landslides by integration of real-time sensors. Geotechnologien Science Report 10: 75-88. Obtenido desde: http://www.winsoc.org/pdf/ATT000 I 0.pdf

Blyth, K. (1997). Floodnet: a telenetwork for acquisition, processing and dissemination of earth observation data for monitoring and emergency management of floods. Hydrological Processes I ( (10): I359- 1375.

Davies, T. (1986). Large debris flows: A macro-viscous phenomenon. Acta Mechanica 63(1): 161.

Hanisch, J., Ergenzinger, P. \& Bonte, M. (2003). Dumpling - An "intelligent" boulder for studying internal processes of debris flows. Proceedings of the Third International Conference on Debris-flow Hazard Mitigation: Mechanics, Prediction, and Assessment, pp. 843-849.

Henderson, T. (2004). Snow Monitoring with Sensor Networks. Annual IEEE Conference on Local Computer Networks, pp. 558-559.

Klingbeil, L. \& Wark, T. (2008). A Wireless Sensor Network for Real-Time Indoor Localisation and Motion Monitoring. 2008 International Conference on Information Processing in Sensor Networks (IPSN), pp. 39-50.

Kwon, Y. \& Agha, G. (2008). Passive Localization: Large Size Sensor Network Localization Based on Environmental Events. 2008 International Conference on Information Processing in Sensor Networks (IPSN), pp. 3-14.

Kwon, O.-H. \& Song, H.-J. (2008). Localization through Map Stitching in Wireless Sensor Networks. IEEE Transactions on Parallel and Distributed Systems 19(1): 93- 105.

Lee, H.-C., Liu, C.-J., Yang, J., Huang, J.-T., Fang, Y.-M., Lee, B.-J. \& King, C.-T. (2008). Using mobile wireless sensors for insitu tracking of debris flows. Proceedings of the 6th ACM 
Conference on Embedded Networked Sensor Systems (SenSys '08), pp. 407-408.

Lee, H.-C., Cho, C.-Y., Fang, Y.-M., Lee, B.-J. \& King, C.-T. (2009a). Exploiting the tradeoff between fast wakeup and long standby in event-monitoring WSN. Proceedings of the 7th ACM Conference on Embedded Networked Sensor Systems (SenSys '09), pp. 403-404.

Lee, H.-C., Lin, C.-Y., Hsu, S.-W. \& King, C.-T. (2009b). On building mobility models for floating objects. Proceedings of the 7th ACM Conference on Embedded Networked Sensor Systems (SenSys '09), pp. 377-378.

Lee, H.-C., Banerjee, A., Fang, Y.-M., Lee, B.-J. \& King, C.-T. (20I0). Design of a Multifunctional Wireless Sensor for In-Situ Monitoring of Debris Flows. IEEE Transactions on Instrumentation and Measurement 59( I I ): 2958-2967.

López, A. (2010, nov. 28) Devastación, dolor y soledad se adueñan de calle Lajas. Al Día. Obtenido de http://www.aldia.cr/ ad_ee/20 I0/noviembre/28/nacionales2603942.html

Murillo, A. (2003, ago. 31). Orosi lucha, con nervios y en alerta. La Nación. Obtenido de http://wrw.nacion.com/In_ee/2003/ agosto/3l/pais3.html

OASIS (2005). Common Alerting Protocol, v. 1.I. Obtenido de: http://www.oasis-open.org/committees/download. php/ I | 35/emergency-CAPv I.I-Corrected_DOM.pdf

Park, J.-G., Demaine, E. D. \& Teller, S. (2008). Moving-Baseline Localization. 2008 International Conference on Information Processing in Sensor Networks (IPSN), pp. I5-26.
Polastre, J., Szewczyk, R. \& Culler, D. (2005). Telos: enabling ultralow power wireless research. 2005 International Symposium on Information Processing in Sensor Networks (IPSN), pp, 364-369. Obtenido de: http://ieeexplore.ieee.org/lpdocs/ epic03/wrapper.htm?arnumber $=\mid 440950$

Ritchie, D. \& Gates, A. (2007). Encyclopedia of Earthquakes and Volcanoes. 3ra ed. New York: Facts on File Science Library.

Ruiz-Sandoval, M., Nagayama, T. \& Spencer, B.F. (2006). Sensor development using Berkeley Mote platform. Journal of Earthquake Engineering 10 (2), 289-309.

Sheth, A., Tejaswi, K., Mehta, P., Parekh, C., Bansal, E., Merchant, S., Singh, T. \& Toyama, K. (2005). Senslide: a sensor network based landslide prediction system. SenSys'05: Proceedings of the 3rd International Conference on Embedded Networked Sensor Systems, pp. 280-281.

Tilak, S., Abu-Ghazaleh, N.B. \& Heinzelman, W. (2002). A taxonomy of wireless micro-sensor network models. ACM SIGMOBILE Mobile Computing and Communications Review 6(2): 28-36.

Vallance, J.W. (2000). Lahars. En: Encyclopedia of Volcanoes. San Diego: Academic Press.

Zostrich Geotechnical (2003). Flash Flood I Debris Flow Warning System. Obtenido el 27 de noviembre de 201I desde: http://www.zostrich.com/Monitoring_PDF/mass_flow_ detector_info.pdf 